\title{
RELIABLE SOFTWARE FRAMEWORK FOR VEHICUlaR SAFETY APPLICATIONS ON ClOUd
}

\author{
Ms. Deepa. D. Saibannavar ${ }^{1}$ and Dr. S. A. Kulkarni ${ }^{2}$ \\ ${ }^{1}$ Lecturer, K.L.S Institute of Management and Research, Belgaum, India \\ ${ }^{2}$ Professor and Head, Dept. of ISE, K.L.S Gogte Institute of Technology, Belgaum, \\ India
}

\begin{abstract}
Vehicular Ad-hoc Networks (VANET'S) have become viable and valuable for their wide variety of novel applications to improve driver's experience. The topology of network is highly time varying due to high mobility of vehicular nodes. This makes challenging to detect and diagnose errors in software applications used in the vehicles. Software reliability in vehicles is critical factor and significant challenge to be met. Misbehaving and faulty software applications in vehicle have to be detected and diagnosed from disrupting operation as it is hard to address in life critical vehicular network environment. The work proposes an advanced diagnostics system to be loaded in Road Side Units (RSU's) so that operating software is periodically transmits the codes generated by the vehicle configured with $O B D$ to the RSU for test. The software is diagnosed at the RSU accessing the data from cloud servers for reliability. Later, a fixed patch is transmitted back to the vehicle via RSU's. The result in this paper shows the analysis of different temperature variables used in vehicles and are efficiently measured.
\end{abstract}

\section{KEYWORDS}

VANET's, Road Side Units, On-Board Units, On-Board Diagnostics, Diagnosis;

\section{INTRODUCTION}

Vehicular Ad-hoc Network (VANET) is an important component part of Intelligent Transportation Systems (ITS). As an open wireless network, VANETs is an application of AdHoc Network for vehicle communication. It mainly consists of the vehicles with on-board units (OBDs), and the roadside units (RSUs). To provide and share information, there are two kinds of communications types one is vehicle-to-vehicle $(\mathrm{V}-2-\mathrm{V})$ communication; the other is vehicleto-roadside communications (V-2-R). By V-2-V communication, people can obtain more information and use the shared information to improve road safety. By V-2-R communication, people can communicate with RSU to access internet for downloading and updating files or inquire neighborhood location information [1]. Thus, compared with the traditional pure infrastructure-based network, the hybrid of $\mathrm{V}-2-\mathrm{V}$ and $\mathrm{V}-2-\mathrm{R}$ communications is promising since it can not only overcome the disadvantages of infrastructure-based network, but also overcome the disadvantage of non-infrastructure-based network.

In Cloud Computing stores information permanently on the internet servers and it allows users to cache it temporarily [2]. The only concern is that the data sending and receiving from cloud must be done reliably. The basic hardware and software are still the integral part but the high level service capabilities are available to build the application. In the background of these services are the data and the resources. 
In recent few years, Vehicular Ad-hoc Networks (VANETs) have gained much interest within the automobile and research worlds. One reason is the consideration in a increasing number of applications designed for passenger safety and comfort- such as emergency braking, traffic jam detection, scope for entertainment and cooperative driving - as well as in applications aiming at the comfort of passengers, such as chat-rooms, games and data-sharing with neighboring vehicles (e.g., CarTorrent[1]) The increased use of software has not only affected car warranty costs but has also made cars harder to repair.

Therefore, this work proposes the remote diagnosis system to detect the errors in software applications to improve reliability of the target vehicle using cloud. The RSU takes the control of the vehicle retrieve log information generated by the OBD device fixed in vehicle and cloud server, testing is performed by comparing the log information retrieved from cloud and vehicle. In this work we make use of "TORQUE Android application" which allows using the codes generated by the OBD. Test results are analyzed, if errors are detected, the results is updated to next RSU to fix the errors and reinstall the correct application software to the vehicle via RSU considering the speed and direction of the vehicle.

Vehicle mobility is usually constrained by road layout, speed limits, traffic and vehicle destination. If the vehicle is travelling within the speed limit the RSU will be able to dynamically reconfigure the correct software or else if it is exceeding the speed limit then warning messages is sent to the driver indicating the error and providing him location of next nearest RSU where he can reconfigure the correct software application. Therefore, this work proposes the diagnosis of the software and software up gradation of software's used by the vehicles on fly.

\section{PREVIOUS WORK}

Safety, comfort driving and infotainment applications for passengers are some of the main services proposed for VANETs. Some services are critical, such as collision warning; other services need bandwidth, such as video-streaming services. QoS (Quality of Service) provisioning and providing highly reliable applications are a challenge in VANETs due to their special features which result in frequent routing path disruptions. In this section, we discuss the related work that has been accomplished in the field of vehicular adhoc networks and also discuss various limitations that were observed.

The authors S. M. Mahmud, S. Shanker, and I. Hossain[3] proposed a protocol by means of which software download is performed using an Intelligent Transportation Systems (ITS) infrastructure. The automotive company issues symmetric keys to encrypt the software transmitted between the software supplier and the vehicle. To increase the security in the transmission, they propose that the software should be sent twice and possibly also in random order to avoid attackers from predicting the message order. To authenticate the vehicle, a set of authentication keys are installed in the vehicle and also stored in a central server and transmitted to the appropriate AP within the ITS during authentication.

The authors I. Hossain and S. M. Mahmud[4] introduced a special device denoted Network Device Monitor (NDM) which is installed in the AP within an ITS infrastructure. The purpose of the NDM is to authenticate vehicles, manage the session keys for the multicast group, and to send software to the vehicles therein. A set of authentication keys are installed in the vehicle and also stored in a central server. These keys are transmitted and used by the NDM to authenticate the vehicle. Furthermore, digital certificates were used as authentication keys for authentication between the automotive company, the software supplier, and the NDM. Nilsson and Larson propose a firmware update process [5] where the firmware is split into smaller fragments and transmitted to the vehicle. Each fragment is hashed and the hash is concatenated to the previous 
fragment. Thus all fragments needs to be hashed before any of them can be transmitted The hash of the first fragment is used as an initial fragment containing a digital signature over the first hash, thereby ensuring that all following hashes cannot be modified without detection. Encryption is also applied to the transmission. This protocol ensures data integrity, data authentication, data confidentiality, and data freshness. Munir Sayyad, Sanjay Nalbalwar, Jagdish Bakal, Shomik Dasgupta [6] proposed Advanced Intelligent Vehicular Analytics records all the vehicular diagnostic data using OBD scan tool program and then wirelessly transmit the data to a central server thereby creating a database of the vehicular data. The database created will be used from the business point of view to generate applications using Web 3.0 architecture. An Interactive Graphical Interface (IGUI) for a cellular phone will be developed so that the owner of a particular vehicle will get real time live update of the vehicle's health. A semantic web of data would be generated to facilitate further progress in this field. This way the owner will know the fault in the vehicle and when to take the vehicle to the service station. The authors in [7] gave the first understanding of the impact of the vehicle's speed, transmission rate, 802.11 bit-rate, and packet size on throughput and delay of vehicle-roadside communication and illustrated a basic picture of how running vehicles contact with roadside hot spots through a "drive-thru" data access.

\section{PROBLEM FORMATION}

To develop a remote diagnosis system to upgrade, test and fix the software application used in vehicles using V-2-I communication model of vehicular ad-hoc networks on cloud. The software in the vehicles (i.e. OBD) can be remotely updated with a bug-fixed software release over the wireless connection and also makes it possible to diagnose many vehicles at the same time via RSU. Reprogramming software in the traditional way is a time consuming procedure that requires test equipment to be connected physically to each vehicle. This increases the driver safety and comfort and allows him to use reliable and updated version of software.

\subsection{Necessity of the proposed system}

Vehicles of today have become gradually more dependent on software to handle their operations and functionalities. For automotive industry maintaining, updating and diagnosing the software in vehicles has therefore become a challenging and costly process. By introducing wireless communications to vehicles, it has greatly improved vehicular maintenance and has brought many other new applications to the vehicles. The following benefits of this system are listed below:

- To provide a new and improved wireless vehicle diagnostic and software upgrade system with a relatively fast response time and improved accuracy.

- To supports Remote software download that will give the customers the opportunity to get the latest software versions installed without having to take the car to an authorized repair shop.

- To support a wide range of applications and services targeting to improve the utilization and the quality of the transportation infrastructure, safety and facilitates driver assistance.

- To help the automotive industry to understand the flaws and problems frequently arising in the vehicles which help them to take appropriate decisions to increase the customer satisfaction that benefits the business.

\subsection{Work process}

This system works as follows:

- Vehicle on road probes the RSU-1 by sending the version number, vehicle ID and hello 
packet. RSU-1(waiting for the hello packet) receives the hello packet and performs the version check accessing its own database.

After the version check performed by the RSU-1 in zone-1 it will reply back with the status (up to date or update required) of the version check to the incoming vehicle (position-2) with OBD as shown in Figure 1. If the received version number of the vehicle matches with version stored on RSU then the RSU will reply the vehicle stating "version up to date". If the received version number of the vehicle does not match with version stored on RSU then the RSU will reply the vehicle stating "Warning: Old version is use Updating is required".

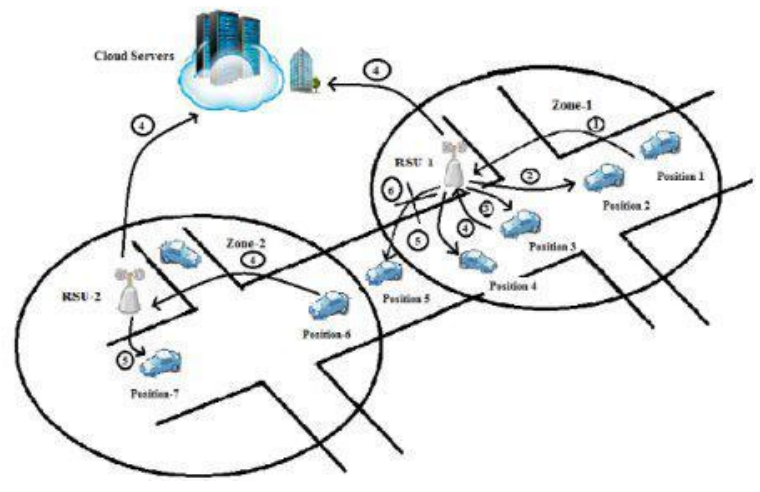

Figure 1. System Infrastructure

- The RSU-1 requests the log information from vehicle (position-3) if the version number is up to date if it not RSU-1 starts updating the application in vehicle (position-3) on successful completion of update a flag is set.

- Vehicle (position-3) will reply back by sending the log information to the RSU-1. At the same the RSU-1 will also access the log details from the cloud server by sending the vehicle ID. The RSU-1 will perform a test by comparing the log details fetched from the vehicle and cloud server.

- If application check is passed in the RSU-1, it will reply back to vehicle (position-4) application working correctly or if it fails the RSU-1 will fix and sends the patch to the vehicle.

- Due to high speed the vehicle (position-5) moves outside the range of Zone-1 link breaks and reliability check is suspended.

- Step 4 is repeated until all the applications are tested. The RSU-2 of Zone-2 resumes testing the reliability of the software of the vehicle once the vehicle enters the Zone-2 (position-6).

- If OBD in vehicle (position-7) successfully receives the file then vehicle responds back to the RSU-2 of Zone-2 by sending the positive Acknowledgement (ACK) and sets the flag bit on successfully completion.

\subsection{Challenges}

To develop this system we come across some of the difficulties

- Vehicles move at higher speeds and consequently lifetimes of communication links are shorter; thus, links must cope with the rapid changes in the network topology.

- Due to the limited factors in OBD such as buffer size, transmission capacity of a RSU and the frequency of data recording. This may led to non-reliable data transfer and also may fail in receiving appropriate data required for diagnosis. 
- Vehicle mobility is usually constrained by road layout, speed limits, traffic and vehicle destination. If the vehicle is in exceeding the speed limit then it results in receiving an incomplete patch. This requires the intelligent file transfer.

- Using of cloud storage is biggest concern due to data integrity and unauthorized Access.

\section{RESULTS}

\subsection{On-Board Diagnostics (OBD)}

In this work the vehicle is fixed with OBD-II device[8]. The OBD-II device provides an access to various subparts of the vehicle. OBD-II collects the data from the various ECU (Engine Control Unit) and sends the data to TORQUE[9] application which will be in form of HEX values. So the TORQUE application takes the responsibility of converting this HEX values to user understandable values which is then used by RSU's for testing and comparing with the details stored on cloud. OBD-II works on Request/Reply basis. The below given figure 2(a) shows how the OBD-II device is connected to vehicle and figure 2(b) is the snapshot of TORQUE application.
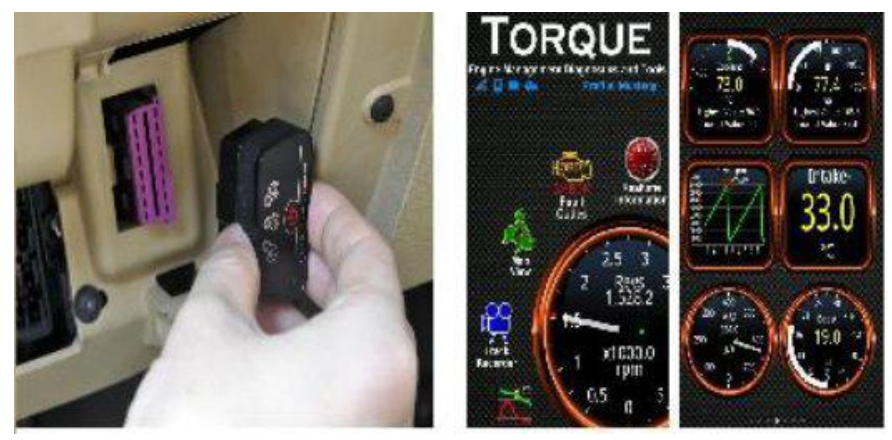

Figure 2 (a) OBD-II (b) TORQUE Application Interface

\subsection{Establishing the communication between vehicles (client) RSU (server) and cloud (backend server):}

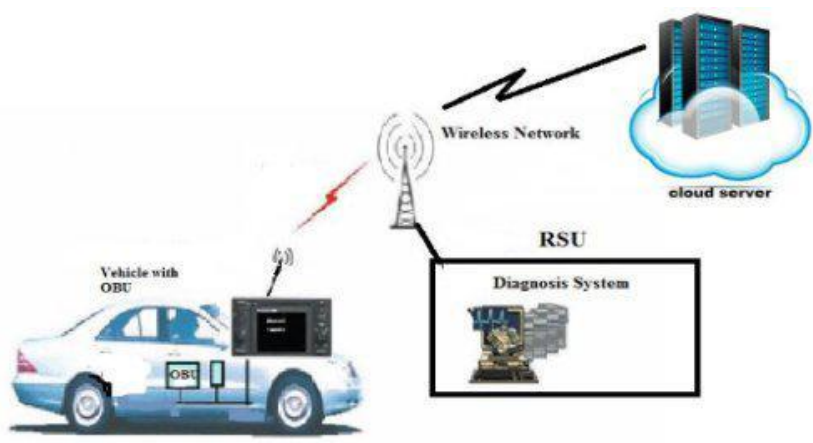

Figure 3. Vehicles with OBD

Wireless connection should be established between the vehicle and RSU and RSU and cloud server. This is done by configuring Ad-hoc network and 802.11d interface cards which allows communicating between the OBD devices installed in the vehicle with the RSU and sending the $\log$ details/codes generated by the application to RSU for testing. Communication between the RSU and the cloud server is through internet as shown in figure 3. Cloud servers update the RSU with the latest applications available, correct threshold values corresponding to different vehicles and so on. 


\subsection{Applications Tested}

Temperature measurement in today's industrial environment encompasses a wide variety of needs and applications. To meet this wide array of needs the process controls industry has developed a large number of sensors and devices to handle this demand. Temperature is a very critical and widely measured variable in all vehicles. Many applications are developed to monitor or control the temperature and warn the drivers regarding the status of devices [10]. In this paper we take an example of application measuring various temperature variables like engine coolant temperature, Air intake temperature and Catalyst temperature. Each of these temperature variables have standard threshold values as shown in Table 1 which defines the status of the engine which can be classified has working in normal condition (operating efficiently or not working as expected (Inefficient operation).

1. Engine Coolant Temperature: The coolant temperature sensor is used to measure the temperature of the engine coolant of an internal combustion engine. The readings from this sensor are then fed back to the Engine control unit (ECU), which uses this data to adjust the fuel injection and ignition timing [11].

2. Air Intake Temperature: The Intake Air Temperature (IAT) monitors the temperature of the air entering the engine [12].

Catalyst Temperature: Catalytic converters are one of the greatest emission add-ons ever to be installed on vehicles [12]. They help in cleaning up the pollutants left over from combustion, they reduce hydrocarbons $(\mathrm{HC})$ and carbon monoxide $(\mathrm{CO})$ emissions to extremely low levels, when everything is operating normally if not the vehicles fail to pass the emission test [12].

Table 1. Temperature measures

\begin{tabular}{|l|l|l|}
\hline \multirow{2}{*}{$\begin{array}{l}\text { Temperature } \\
\text { variables }\end{array}$} & \multicolumn{2}{|l|}{ Threshold temperature Values } \\
\cline { 2 - 3 } Engine Coolant & Operating Effiecintly & Inefficient operation \\
Temperature & Range $200 \circ \mathrm{F}-225 \circ \mathrm{F}$ & $225 \circ \mathrm{F}$ Above \\
\hline $\begin{array}{l}\text { Intake Air } \\
\text { Temperature }\end{array}$ & $+40 \circ \mathrm{F} /-40 \circ \mathrm{F}$ & $+40 \circ \mathrm{F}-40 \circ \mathrm{F}$ Above \\
\hline Catalyst & Ambient Temperature & Ambient Temperature \\
Temperature & Range $400 \circ \mathrm{F}-1600 \circ \mathrm{F}$ & $1900 \circ \mathrm{F}$ Above \\
\hline
\end{tabular}

The graph in Figure 4 performs the analysis of Engine Coolant temperature against the speed of the vehicle for low temperature, high temperature and required temperature. The Graphs shown in figure 4(a)(b)(c) is generated in Java[13] by feeding the real time data obtained by TORQUE application. The graph in Figure 4 performs the analysis of Intake Air Temperature vs. speed also considering constant ambient temperature. The graph in Figure 5 performs the analysis of Catalyst Temperature vs. speed also considering constant ambient temperature.

\subsection{Log Section}

Log section of the application running displays the current status of application test as well as it also displays the connection status with RSU. It gives the following details

1. Connection establishment with the nearest RSU.

2. Version check performed by the RSU.

3. Updation process scheduled by the RSU if current version in use is old.

4. Status of working of application i.e the application is displaying appropriate messages or no if yes then working is fine else it displays application is faulty. 
Therefore it should schedule the updation.

5. Finally it also displays which all applications have successfully updated so that it can schedule the application only which has not been updated. Snapshot of log section is shown in figure 5 .

\subsection{Cloud Server}

Cloud server stores all the details of the applications running in the vehicles like the latest version release of applications, which are the suitable application available to vehicles based on the OBD model so that it can update the RSU's frequently as soon as the new version is available. It runs in background and uses PHP scripts to fetch the data of the vehicle.
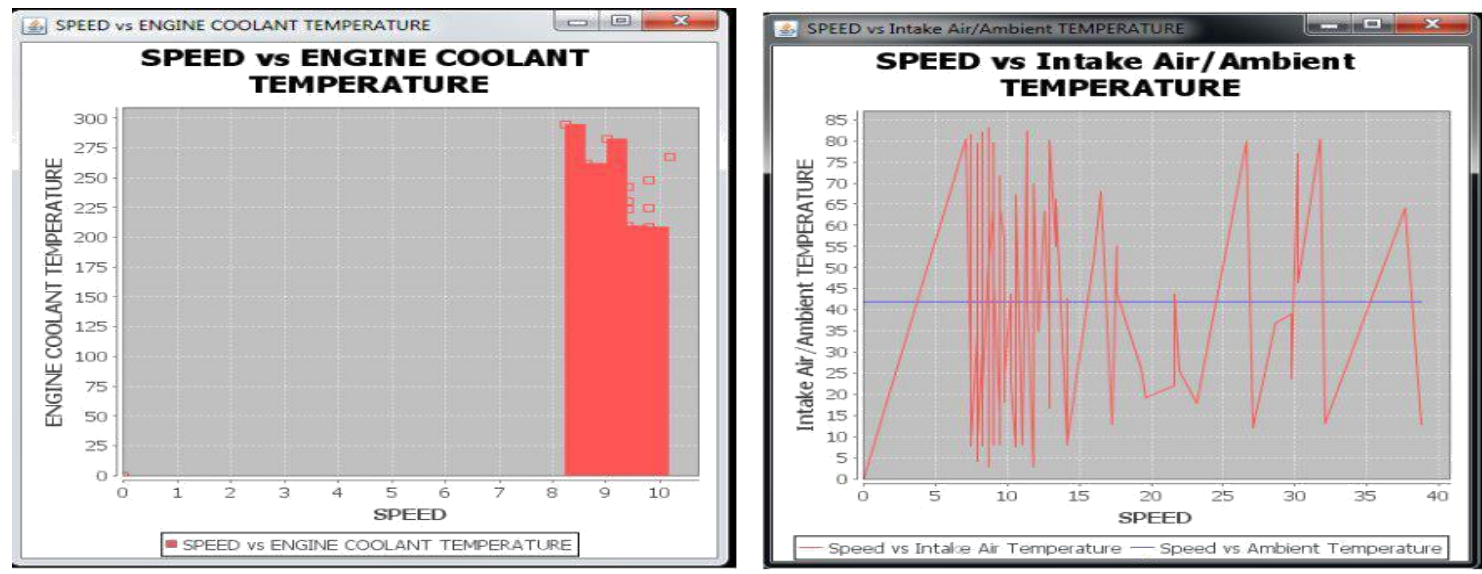

Figure 4 (a) Engine coolant temperature vs. Speed (b) Intake air temperature vs Speed

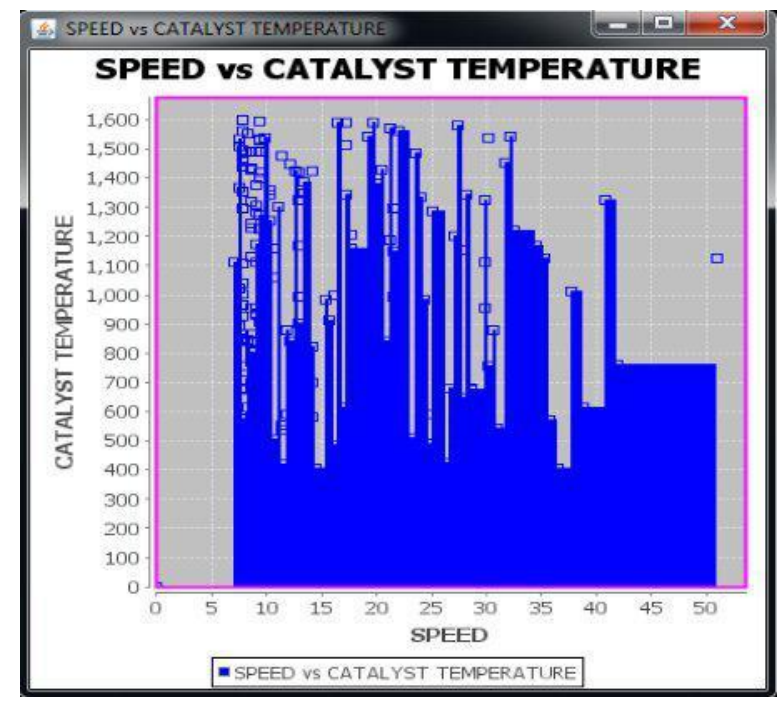

Figure 4 (c) Intake air temperature vs Speed

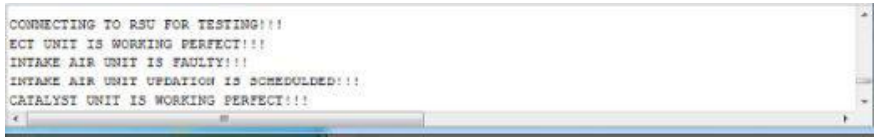

Figure 5. Log section

In this paper, the proposed system checks whether the application developed to give the warning or alert messages to driver when the temperature is exceeding the threshold value is operating or 
International Journal on Cybernetics \& Informatics (IJCI) Vol. 5, No. 1, February 2016

is displaying appropriate warning/alert messages. It also checks the application installed is a latest version or require the application updating. If the application is not displaying appropriate messages then the bug in the application has to be detected by using the values generated by the vehicle which is sent to RSU and the RSU performs the testing by requesting the application details from the cloud server and fix the bug by reinstalling a bug free patch to the vehicle.

\section{CONCLUSION}

The convergence of wireless telecommunications and various kinds of services are enabling the deployment of different kinds of application software's in vehicles to improve the driver comfort and safety. Reliability of software applications in vehicles is very critical so these have to be periodically tested and diagnosed. This paper presents a new software diagnosis system which also updates the software applications installed in stationary vehicles on fly. The results in this paper are performed by simulating the temperature testing application which warns the driver about faulty behavior of engine by checking the temperature variables and helps to test and diagnose software applications in vehicles to improve the reliability and update them if required with the help of the cloud servers. In future this approach can be used for testing few more applications to provide bug free applications to improve driver safety and comfort.

\section{REFERENCES}

[1] Zhang Jianhong, Xu Min, and Liu Liying, "On the security of a secure batch verification with Group Testing for VANET”, International Journal of Network Security, Vol.16, No.5, PP.351-358, Sept. 2014

[2] "Privacy-Preserving public auditing for Data Storage security in Cloud ComputingCong" Wang, Qian Wang, and Kui Ren Wenjing Lou "Technical Program at IEEE INFOCOM 2010

[3] Mathias Johanson Alkit Communications AB Sweden "Information and communication support for Automotive testing and validation" New Trends and Developments in Automotive System Engineering

[4] S. M. Mahmud, S. Shanker, and I. Hossain. "Secure software upload in an intelligent Vehicle via wireless communication links". Proceedings of the IEEE Intelligent Vehicles Symposium. 2005, pp. 588-593. doi: 10.1109/IVS.2005.1505167

[5] I. Hossain and S. M. Mahmud. "Secure multicast protocol for remote software upload in intelligent Vehicles". Proc. of the 5th Ann. Intel. Vehicle Systems Symp. of National Defense Industries Association (NDIA). National Automotive Center and Vectronics Technology. Traverse City, Michigan, June 2005, pp. 145-155

[6] D. K. Nilsson and U. E. Larson. "Secure Firmware Updates over the Air in Intelligent Vehicles". Proceedings IEEE International Conference on Communications Workshops (ICC Workshops '08). May 2008, pp. 380-384. doi: 10.1109/ICCW.2008.78

[7] Munir Sayyad1*, Sanjay Nalbalwar2, Jagdish Bakal3, Shomik Dasgupta "Advanced intelligent Vehicular analytics (AIVA) using IMS Architecture", International Journal of Smart Home Vol. 5, No. 2, April, 2011.

[8] OBD-II Background http://www.obdii.com/background.html

[9] TORQUE Engine Performance and Diagnostic Tool http://torque-bhp.com/

[10] MVFRI reasearch Summary Kennerly H. Digges, Edmund Fournier Underhood Temperature Measurements Based on a contract with: Biokinetics and Associates, Ltd.

[11] Engine coolant temperature ECT sensor engine codecom. ] http://carrelease.info/view/engine-coolanttemperature-ect-sensor-engine-codescom.

[12] catalytic converter-Auto diagnosis repair help http://www.aa1car.com/library/converter.htm

[13] Socket Communications http://www.oracle.com/technetwork/java/socket-140484.html 